\title{
HD 219134 Revisited: Planet d Transit Upper Limit and Planet f Transit Nondetection with ASTERIA and TESS
}

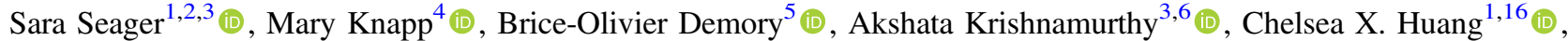

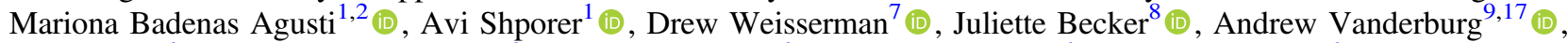 \\ Matthew Smith ${ }^{6}$ (10), Christopher M. Pong ${ }^{6}$, Vanessa P. Bailey ${ }^{6}\left(\mathbb{D}\right.$, Amanda Donner ${ }^{6}$, Peter Di Pasquale ${ }^{6}$, Brian Campuzano ${ }^{6}$, \\ Colin Smith ${ }^{6}$, Jason Luu ${ }^{6}$, Alessandra Babuscia ${ }^{6}$, Robert L. Bocchino, Jr. $^{6}$, Jessica Loveland ${ }^{6}$, Cody Colley ${ }^{6}$, Tobias Gedenk ${ }^{4}$, \\ Tejas Kulkarni ${ }^{6}$, Kyle Hughes ${ }^{6}$, Mary White ${ }^{6}$, Joel Krajewski ${ }^{6}$, Lorraine Fesq ${ }^{6}$, George Ricker ${ }^{1}$ (i), Roland Vanderspek ${ }^{1}$ (i),

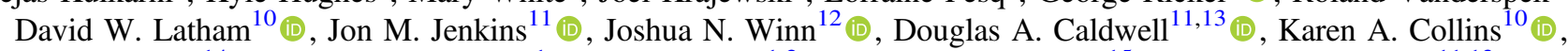

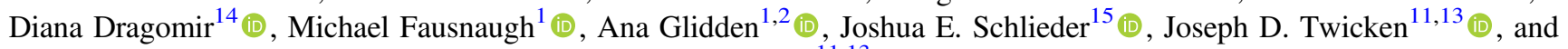 \\ Bill Wohler ${ }^{11,13}$ (1) \\ ${ }^{1}$ Department of Physics and Kavli Institute for Astrophysics and Space Research, Massachusetts Institute of Technology, Cambridge, MA 02139, USA \\ seager@mit.edu \\ ${ }^{2}$ Department of Earth, Atmospheric, and Planetary Sciences, Massachusetts Institute of Technology, 77 Massachusetts Avenue, Cambridge, MA 02139, USA \\ ${ }^{3}$ Department of Aeronautics and Astronautics, Massachusetts Institute of Technology, Cambridge, MA 02139, USA \\ ${ }^{4}$ Haystack Observatory, Massachusetts Institute of Technology, Cambridge, MA 02139, USA \\ ${ }^{5}$ Center for Space and Habitability, University of Bern, Gesellschaftsstrasse 6, CH-3012 Bern, Switzerland \\ ${ }^{6}$ Jet Propulsion Laboratory, California Institute of Technology, 4800 Oak Grove Drive, Pasadena, CA 91109, USA \\ ${ }^{7}$ Department of Astronomy, University of Michigan, Ann Arbor, MI 48109, USA \\ ${ }^{8}$ Division of Geological and Planetary Sciences, Caltech, Pasadena, CA, USA \\ ${ }^{9}$ Department of Astronomy, The University of Texas at Austin, Austin, TX 78712, USA \\ ${ }^{10}$ Center for Astrophysics | Harvard \& Smithsonian, 60 Garden Street, Cambridge, MA 02138, USA \\ ${ }^{12}$ Department of Astrophysical Sciences, Princeton University, 4 Ivy Lane, Princeton, NJ 08544, USA

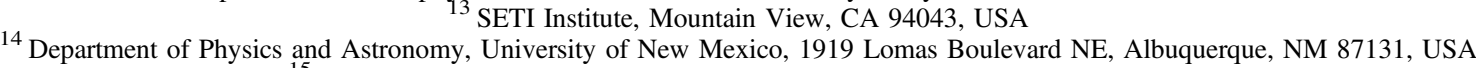 \\ ${ }^{15}$ NASA Goddard Space Flight Center, Code 667, Greenbelt, MD 20771, USA \\ Received 2020 May 17; revised 2020 November 14; accepted 2020 November 22; published 2021 February 12
}

\begin{abstract}
HD 219134 is a K3V dwarf star with six reported radial-velocity discovered planets. The two innermost planets b and $\mathrm{c}$ show transits, raising the possibility of this system to be the nearest $(6.53 \mathrm{pc})$, brightest $(V=5.57)$ example of a star with a compact multiple transiting planet system. Ground-based searches for transits of planets beyond $\mathrm{b}$ and $\mathrm{c}$ are not feasible because of the infrequent transits, long transit duration $(\sim 5 \mathrm{hr})$, shallow transit depths $(<1 \%)$, and large transit time uncertainty ( $\sim$ half a day). We use the space-based telescopes the Arcsecond Space Telescope Enabling Research in Astrophysics (ASTERIA) and the Transiting Exoplanet Survey Satellite (TESS) to search for transits of planets $\mathrm{f}\left(P=22.717\right.$ days and $\left.M \sin i=7.3 \pm 0.04 M_{\oplus}\right)$ and $\mathrm{d}\left(P=46.859\right.$ days and $\left.M \sin i=16.7 \pm 0.64 M_{\oplus}\right)$. ASTERIA was a technology demonstration CubeSat with an opportunity for science in an extended program. ASTERIA observations of HD 219134 were designed to cover the $3 \sigma$ transit windows for planets f and d via repeated visits over many months. While TESS has much higher sensitivity and more continuous time coverage than ASTERIA, only the HD $219134 \mathrm{f}$ transit window fell within the TESS survey's observations. Our TESS photometric results definitively rule out planetary transits for HD $219134 \mathrm{f}$. We do not detect the Neptune-mass HD $219134 \mathrm{~d}$ transits and our ASTERIA data are sensitive to planets as small as $3.6 R_{\oplus}$. We provide TESS updated transit times and periods for HD $219134 \mathrm{~b}$ and c, which are designated TOI 1469.01 and 1469.02 respectively.
\end{abstract}

Unified Astronomy Thesaurus concepts: Exoplanet astronomy (486); Broad band photometry (184)

\section{Introduction}

\subsection{The HD 219134 Planetary System}

HD 219134 is a bright, nearby K3V star reported to host six radial-velocity discovered exoplanets (Motalebi et al. 2015; Vogt et al. 2015; Johnson et al. 2016; Gillon et al. 2017). The two innermost planets HD $219134 \mathrm{~b}$ and c, with periods of 3.1 and 6.8 days respectively, were found to transit with Spitzer (Motalebi et al. 2015; Gillon et al. 2017). For planetary system parameters see Table 1 and Figure 1. HD 219134 is the nearest and brightest star presently known to host transiting planets (6.53 pc, Gaia Collaboration et al. 2018; Stassun et al. 2018;

\footnotetext{
${ }^{16}$ Juan Carlos Torres Fellow.

${ }^{17}$ NASA Sagan Fellow.
}

and $V=5.57$, Oja 1993), making HD $219134 \mathrm{~b}$ and $\mathrm{c}$ eminently well suited for follow-up atmospheric characterization. HD 219134, also known as TOI 1469, has a stellar radius of $0.778 \pm 0.005 R_{\odot}$ (Boyajian et al. 2012) and stellar mass of $0.81 \pm 0.03 M_{\odot}$ (Gillon et al. 2017), with R.A. $=23: 13: 16.780$ and decl. $=+57: 10: 10.05$ (J2015.5). For other stellar parameters see the Appendix.

The HD 219143 planetary system has promise to be the nearest, brightest example of a compact multiple-planet transiting system-over 100 times closer and more than 200 times brighter than the canonical Kepler 11 system (Lissauer et al. 2011). Indeed, even with periods as long as 22.717 and 46.859 days for planets $\mathrm{f}$ and $\mathrm{d}$ respectively, the planets still have a fairly high transit probability owing to the transiting 
Table 1

Parameters for HD 219134 Planets b, c, f, and d

\begin{tabular}{lccrrr}
\hline \hline Planet & $P(\mathrm{~d})$ & $a(\mathrm{au})$ & $M\left(M_{\oplus}\right)$ & $R\left(R_{\oplus}\right)$ & $e$ \\
\hline $\mathrm{b}$ & $3.092926 \pm 0.000010$ & $0.03876 \pm 0.00047$ & $4.74 \pm 0.19$ & $1.602 \pm 0.055$ & $0.511 \pm 0.047$ \\
$\mathrm{c}$ & $6.76458 \pm 0.00033$ & $0.06530 \pm 0.00080$ & $4.36 \pm 0.22$ & $0.062 \pm 0.039$ \\
$\mathrm{f}$ & $22.717 \pm 0.015$ & $0.1463 \pm 0.0018$ & $>7.30 \pm 0.40$ & $\cdots$ & $0.148 \pm 0.047$ \\
$\mathrm{~d}$ & $46.859 \pm 0.028$ & $0.2370 \pm 0.0030$ & $>16.17 \pm 0.64$ & $\cdots$ & $0.138 \pm 0.025$ \\
\hline
\end{tabular}

Note. $P$ is period in days, $a$ is semimajor axis in astronomical units, $M_{\oplus}$ is planet mass in Earth masses, $R_{\oplus}$ is planet radius in Earth radii, and $e$ is orbital eccentricity. Planet masses for $\mathrm{f}$ and $\mathrm{d}$ are minimum masses, hence the $>$ sign. Planets $\mathrm{f}$ and $\mathrm{d}$ (and g, not shown) are less firm than planets $\mathrm{b}$ and $\mathrm{c}$ due to their orbital periods' proximity to the stellar rotation period (45.6 days) or its harmonics (Johnson et al. 2016; Folsom et al. 2018). Data from Gillon et al. (2017). For updated $P$ for planets $\mathrm{b}$ and $\mathrm{c}$, see Table 3 .

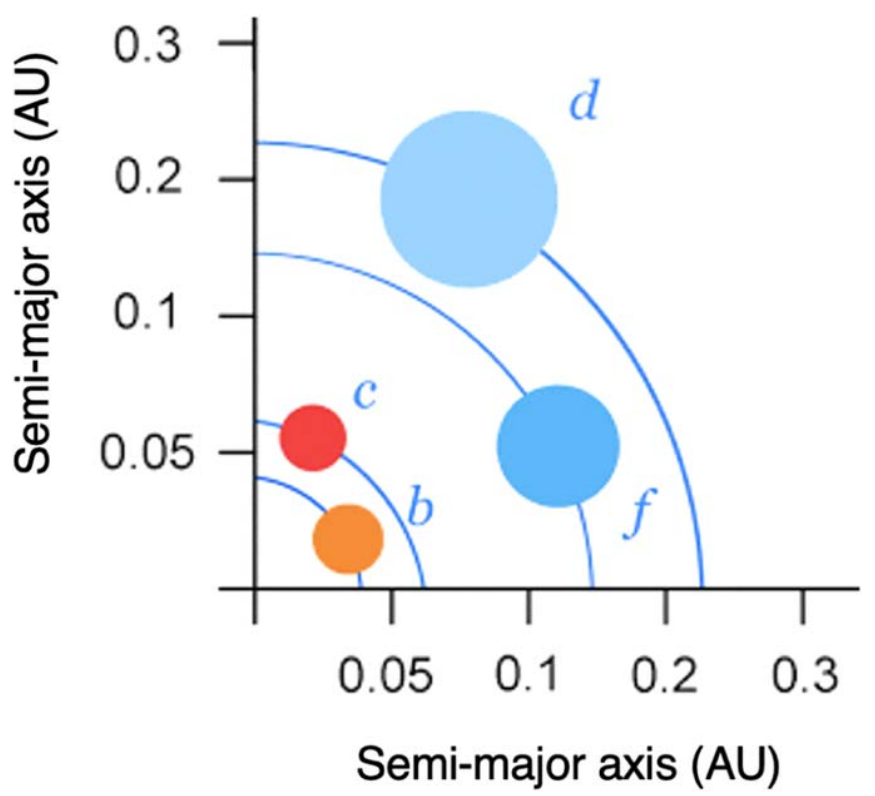

Figure 1. Architecture of the inner HD 219134 planetary system. Four of the six reported planets are shown with their approximate relative sizes. Planets $b$ and $\mathrm{c}$ have measured radii based on their transits. The radii of planets $\mathrm{d}$ and $\mathrm{f}$ are assigned from planets with similar masses. Exterior planets $\mathrm{g}$ and $\mathrm{h}$ are not shown, because ASTERIA did not obtain measurements during their potential transit windows. Figure adapted from: http://backalleyastronomy.blogspot. com/2017/04/.

geometry of planets $\mathrm{b}$ and $\mathrm{c}$. Assuming coplanarity of planets $\mathrm{f}$ and $\mathrm{d}$ with planets $\mathrm{b}$ and $\mathrm{c}$, the a posterior transit probabilities of planets $\mathrm{f}$ and $\mathrm{d}$ are $13.1 \%$ and $8.1 \%$, respectively (Gillon et al. 2017).

The detections of planets HD $219134 \mathrm{f}, \mathrm{d}$, and $\mathrm{g}$ are less firm than planets $b$ and $c$, owing to the proximity of their orbital periods to the stellar rotation period (45.6 days) or its harmonics (Johnson et al. 2016; Folsom et al. 2018). While it is not unexpected to see a $2: 1$ period ratio between planets (e.g., Winn \& Fabrycky 2015), it is concerning that the periods of planets $\mathrm{f}, \mathrm{d}$, and $\mathrm{g}$ happen to be at $1 / 2,1$, and 2 times the stellar rotation period.

A ground-based search for transits of planets HD $219143 \mathrm{f}$ and $\mathrm{d}$ is impractical because of the low frequency and long duration of the transits and the ephemeris uncertainty (see parameters in Table 2). The long periods (approximately 23 and 47 days) mean that the number of transit events per observing season is small, making observable transit events from a single ground-based location rare. The combination of the relatively long transit duration $(3.8 \mathrm{hr}$ for planet $\mathrm{f}$ and $5.6 \mathrm{hr}$
Table 2

HD $219134 \mathrm{f}$ and d Midtransit Times and Other Parameters Used in Our Planet Search. Values from Gillon et al. (2017)

\begin{tabular}{|c|c|c|}
\hline Planet Parameter & Planet $\mathrm{f}$ & Planet d \\
\hline Transit Duration (hr) & $<3.82 \pm 0.22$ & $<5.62 \pm 0.17$ \\
\hline Transit Depth (ppm) & $>236 \pm 8$ & $>358 \pm 7$ \\
\hline Planet Radius $\left(R_{\oplus}\right)$ & $>1.31 \pm 0.02$ & $>1.61 \pm 0.02$ \\
\hline Midtransit Time (BJD & $7,716.31 \pm 0.50$ & $7,726.03 \pm 0.63$ \\
\hline
\end{tabular}

Note. Planet radii lower limits are based on the extreme case of a pure iron planet. For updated $T_{0}$, see Table 3.

for planet d) with the uncertainty on the predicted transit time (on the order of half a day, longer than the anticipated transit duration itself) means that it is both difficult and unlikely to catch an entire transit from a single ground-based location. Catching only the ingress or egress makes it difficult to be confident in the detection of the flux decrease or increase. The issues are exacerbated by the shallow transits expected to be roughly $0.1 \%$ deep. This small transit depth is at or beyond the typical capabilities of $1 \mathrm{~m}$ class ground-based telescopes, the telescopes that are commonly being used for this purpose, even under ideal conditions such that the HD $219134 \mathrm{f}$ and d transits are expected to be shallow with amplitude similar to systematic noise signals that are known to plague ground-based light curves. We note that despite a shallow transit depth of $0.13 \% \pm 0.02 \%$, NGTS-4b was discovered with a single $20 \mathrm{~cm}$ aperture telescope, enabled by a combined 28 transits for the 1.34 day orbit with a 272 night baseline (West et al. 2019).

To search for transits of HD $219134 \mathrm{~d}$ and $\mathrm{f}$ we used two complementary space-based photometric data sets. The data set from the Arcsecond Space Telescope Enabling Research in Astrophysics (ASTERIA; Smith et al. 2018) has targeted observations with extensive coverage of the transit windows, while the data set from the Transiting Exoplanet Survey Satellite (TESS; Ricker et al. 2015) has better photometric precision.

\subsection{ASTERIA}

ASTERIA was a small space telescope $(10 \mathrm{~cm} \times 20 \mathrm{~cm}$ $\times 30 \mathrm{~cm}, 12 \mathrm{~kg}$, a "6U CubeSat") deployed from the International Space Station in 2017 November into a low-Earth orbit. The spacecraft was operational until 2019 December and reentered Earth's atmosphere in late 2020 April. ASTERIA's refractive camera had a $6 \mathrm{~cm}$ aperture, $85 \mathrm{~mm}$ focal length, a bandpass from $500 \mathrm{~nm}$ to $900 \mathrm{~nm}$, a plate scale of 15 !" 8 per pixel, and a field of view of $11^{\circ} .2 \times 99^{\circ} .6$. 
ASTERIA was a technology demonstration mission to prove precise pointing and detector thermal control are possible in a small package, with an eye toward enabling high photometric precision. ASTERIA successfully met its requirements by achieving the following performance goals: reaching line-ofsight pointing stability of approximately $0 . / 5 \mathrm{rms}$ over 20minute observations, pointing repeatability of 1 mas rms from one observation to the next, and focal plane temperature stability better than $\pm 0.01 \mathrm{~K}$ over 20 -minute observations (Pong 2018; Smith et al. 2018). Developed at the Jet Propulsion Laboratory (JPL) in collaboration with the Massachusetts Institute of Technology (MIT), ASTERIA traces its roots back to the ExoplanetSat mission concept developed by MIT and Draper Laboratory (e.g., Smith et al. 2010; Pong et al. 2010). For details of the ASTERIA development, flight system, ground system, on-orbit operation, and on-orbit performance see Smith et al. (2018) and Knapp et al. (2020).

ASTERIA used a complementary metal-oxide semiconductor (CMOS) array detector instead of the traditional chargecoupled device (CCD) detector. We chose a CMOS detector primarily for its fast read out $(20 \mathrm{~Hz}$; necessary for the fine pointing control algorithm), its ability to operate at room temperature, and its relative insensitivity to radiation damage. CMOS detectors can be operated at room temperature, as they generate ultra-low dark current $(20 \mathrm{e}-/ \mathrm{pixel} / \mathrm{s})$ compared to 250-500 e-/pixel/s generated by CCDs at the same temperature. Hence, CMOS detectors do not require passive or active cooling systems that would otherwise add significantly to the mass and size of the spacecraft. Despite these distinct advantages, CCDs have traditionally dominated space-based imaging and spectroscopy due to their sensitivity via high quantum efficiency.

ASTERIA was not designed to deliver precise photometry, even though its underlying precise pointing and thermal control are enabling technologies for highly precise photometry. Nonetheless, ASTERIA was able to detect the transit of $55 \mathrm{Cnc}$ e, a planet nearly twice the radius of Earth transiting a Sun-sized star with a transit depth of approximately $400 \mathrm{ppm}$ (Knapp et al. 2020). After completion of its 90 day prime mission, ASTERIA was in part used for opportunistic science, including observations of the bright nearby stars Algol, Alpha Centauri A and B, 55 Cancri, and HD 219134.

\subsection{TESS}

TESS is an MIT-led, NASA Medium-class Explorer Mission that launched in 2018 April (Ricker et al. 2015). TESS carries four identical, specialized wide-field CCD cameras, each covering $24^{\circ}$ x $24^{\circ}$ on the sky with a $10 \mathrm{~cm}$ aperture, a red bandpass of $600-1000 \mathrm{~nm}$, and plate scale of $21^{\prime \prime}$ per pixel. TESS is in an innovative high-Earth lunar resonance orbit (Gangestad et al. 2013) and a highly inclined, highly elliptical orbit in resonance with the moon, giving TESS a relatively unobstructed, continuous orbit night observations in a thermally stable environment.

During its two-year prime mission, TESS surveyed $70 \%$ of the sky, by monitoring each $24^{\circ} \times 96^{\circ}$ strip of the sky (called a Sector) for two orbits, or 27.4 days. TESS surveyed the southern ecliptic hemisphere in the first year of its prime mission and will complete the northern ecliptic hemisphere during its second and final year of its prime mission in 2020 June.
TESS is anticipated to find thousands of exoplanets during its prime mission (Sullivan et al. 2015; Barclay et al. 2018; Huang et al. 2018), most with orbital periods less than one month. Although TESS will not be able to discover true Earth analogs (that is, Earth-sized exoplanets in 365 day period orbits about Sun-like stars), TESS will be capable of finding Earthsized and super-Earth-sized exoplanets (where super-Earths are loosely defined as planets with radii up to 1.7 times Earth's radius) transiting $\mathrm{M}$-dwarf stars, stars that are significantly smaller and cooler than our Sun. One of the TESS Level One mission requirements is to deliver 50 small $\left(R_{p}<4 R_{\oplus}\right)$ planets with measured masses to the community.

During TESS' survey of the sky, TESS naturally monitors many known transiting planet-hosting stars, including HD 219134.

In this paper we proceed with a description of our observations and photometric methods in Section 2, present our transit nondetection results and discussion in Section 3, and conclude with a summary in Section 4.

\section{Observations and Methods}

\subsection{ASTERIA Observations}

ASTERIA orbited Earth approximately once every 90 minutes, with orbit night typically lasting between 20 and 35 minutes. ASTERIA only observed during orbit night, therefore observations of HD 219134 were not continuous. Furthermore, because of its small aperture, data from different observing sequences must be phase-folded and binned in order to reach the photometric precision needed to detect shallow transits. ASTERIA's observational constraints (e.g., Sun, Moon, and Earth limb exclusion angles) and operational constraints (e.g., spacecraft related factors) are described in detail in Knapp et al. (2020).

We scheduled ASTERIA observations of HD 219134 (see the Appendix) to target the predicted transit windows of planets $f$ and d (Table 2 with values from Gillon et al. 2017). The transit window predictions take into account uncertainty in the midtransit time $T_{0}$ and other parameters fit in Gillon et al. (2017). The orbital periods of planets $f$ and $d$ are close to 2:1 (see Table 1), so their predicted transits occur close to one another. The first targeted campaign (so08 through so12, where "so" means science observation) spanned the predicted transit of HD $219134 \mathrm{f}$ (midpoint 2018 March 15) and HD $219134 \mathrm{~d}$ (midpoint 2018 March 17). The second campaign (ex11, so18, where "ex" means experimental observation) targeted planet $\mathrm{f}$ only (midpoint 2018 April 7). Observations during this campaign were affected by unfavorable geometry between the spacecraft and the star, resulting in short duration observations. The third campaign (so21, so22) targeted the f transit primarily, with some overlap with the beginning of the $d$ transit window. The fourth campaign (so33 through so35) targeted both planets' transit windows (2018 June). Campaign five (2018 July, so37, so38) targeted the transit window of HD $219134 \mathrm{f}$. The final two campaigns in 2018 August and September (so39 through so48) were focused on covering the full $3 \sigma$ transit window for planet $\mathrm{d}$, with incidental coverage of planet $\mathrm{f}$ transit windows. Other opportunistic observations were carried out when science time was available and spacecraft viewing geometry with HD 219134 was favorable.

For a visual representation of the HD 219134 observing sequences of ASTERIA see Figure 2. For transit coverage of 

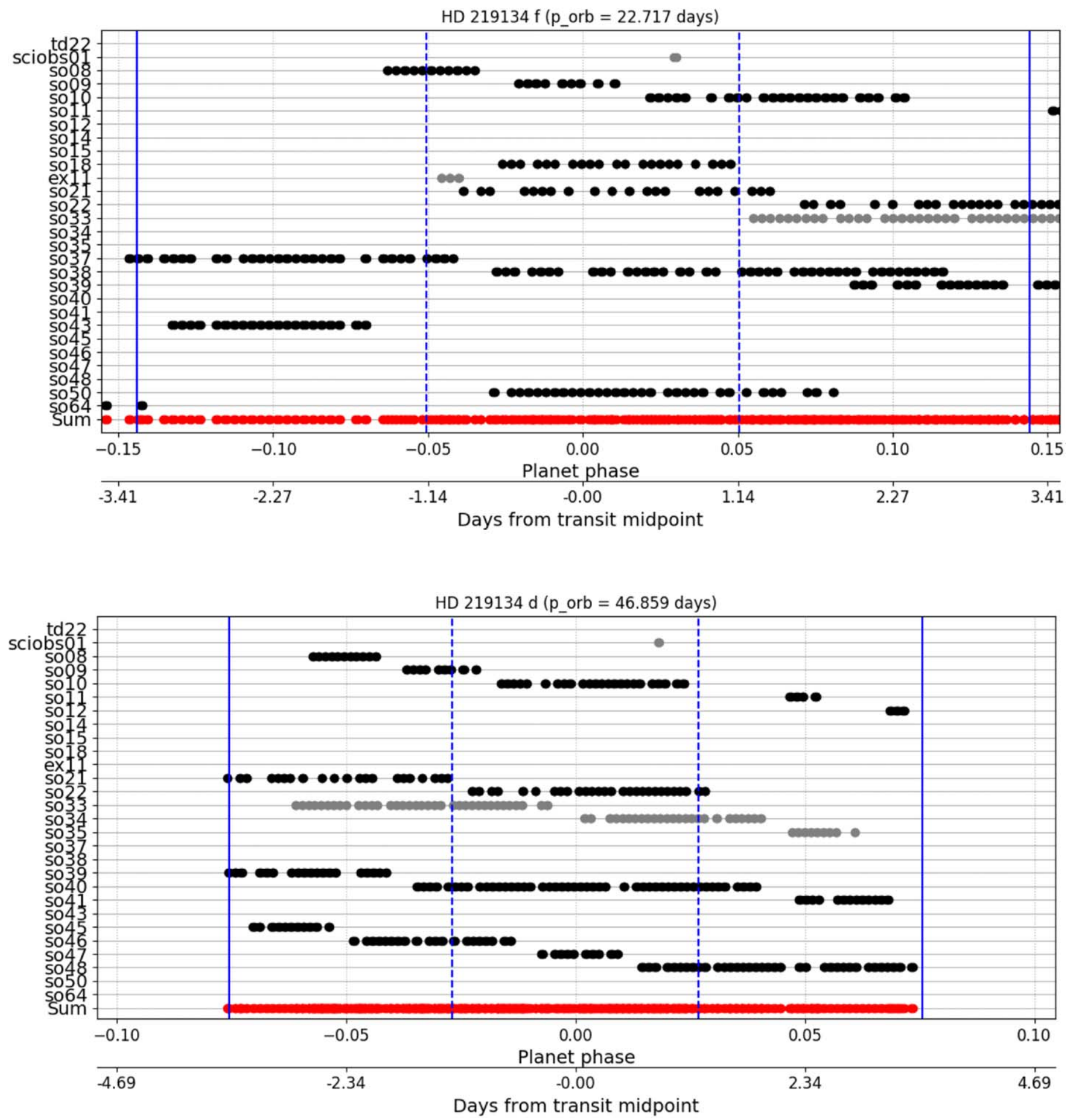

Figure 2. ASTERIA HD 219134 observations. The $x$-axis shows phase, with phase $=0$ corresponding to the predicted transit midpoint. A second horizontal axis shows days from the transit midpoint. The $y$-axis shows different observing sequences (defined in the Appendix), with each black point corresponding to an orbit night. Gray points indicate observations that were not used in the analysis presented in this paper. The red points and bars at the bottom of the plot represent the union of the black points above. The vertical dashed blue lines show the $1 \sigma$ transit window as of 2019 March 28 ; the solid blue bars represent the $3 \sigma$ transit window for the same date. Top: HD 219134 f. Bottom: HD 219134 d.

HD 219134 in the form of data density per planet phase see the Appendix.

\subsection{ASTERIA Data Analysis}

Here we provide a summary of our data reduction pipeline, with full details described in Knapp et al. (2020).

\subsubsection{ASTERIA CMOS Detector and Calibration Frames}

ASTERIA was the first space telescope to attempt science using CMOS detectors. The main difference between CMOS and CCD detectors for precise photometry is that the CMOS readout is parallelized column-wise whereas the pixels of a CCD detector are read out at a single point. For the CMOS 


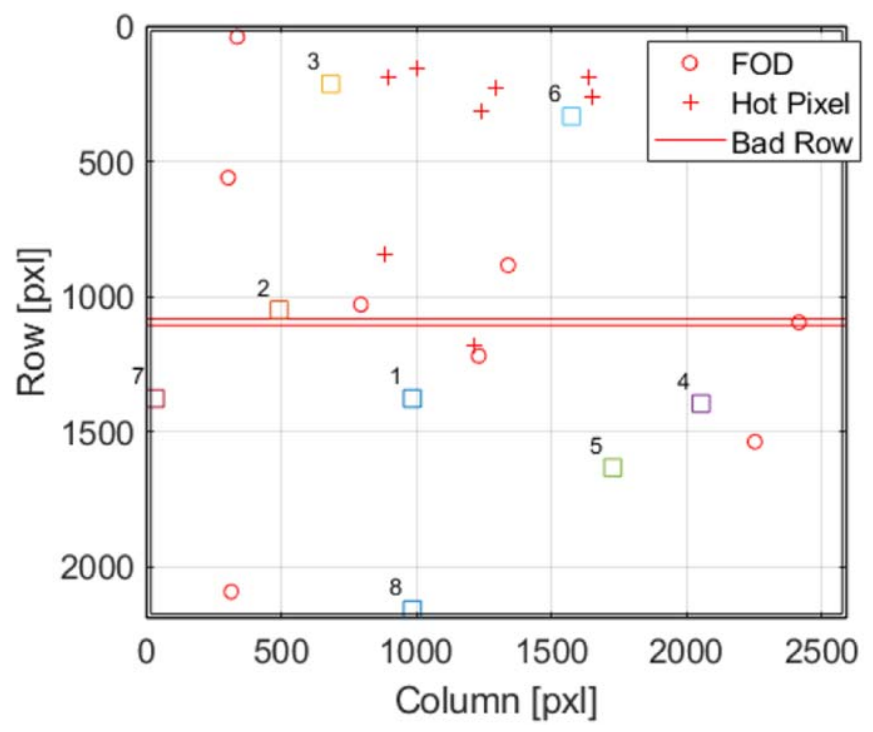

Figure 3. Detector setup for ASTERIA HD 219134 observations. The target star, HD 219134, is centered in window 1. Window 8 served as the calibration window for window 1. The additional windows contain guide stars for ASTERIA's fine pointing algorithm. Known flaws and foreign object debris (FOD) on the detector are also noted.

detector, each pixel has a different amplifier and each column has a different analog-to-digital converter (ADC). Gains and offsets are therefore column-dependent. The systematics for the ASTERIA CMOS are not as spatially uniform compared to a $\mathrm{CCD}$, because the readout electronics vary column to column.

Due to ASTERIA's status as a technology demonstration mission, there was no requirement or opportunity to characterize the detector in-depth, take calibration data, or validate the photometric performance prior to launch. This affected our data reduction pipeline because we have no ground-based dark frames or flat frames from pre-flight laboratory testing. ASTERIA's camera had no shutter so we could not obtain space-based darks, although dark frames are ultimately not needed for ASTERIA data reduction due to the short $50 \mathrm{~ms}$ integration time. ASTERIA did acquire approximated flat frames in space by purposely pointing the telescope in such a way that stray sunlight illuminated the detector. See Knapp et al. (2020) for more details.

Bias was removed from light images column-wise through the use of calibration windows collected contemporaneously with light windows. The calibration window was placed at the bottom edge of the detector where there are both electrically dark pixels (electrically tied to the ground so that photoelectric charge cannot accumulate) and separate optically dark pixels (blocked from light exposure) on the same columns as the light window. We used the electrically dark pixels for the bias correction. See Figure 3, where HD 219134 is centered in window 1 and window 8 is the calibration window.

\subsubsection{ASTERIA Data Reduction}

Windowed images from ASTERIA observations were downlinked to the operations center at JPL and then processed into FITS files using custom software. The image data included contemporaneous calibration windows chosen from the edge of the detector (Figure 3). Metadata, including spacecraft housekeeping telemetry, were downlinked separately from the images and then included in the FITS header. Each FITS image contains a 1-minute integration that resulted from the onboard coadding of 1200 individual very short exposure $(50 \mathrm{~ms})$ frames. Individual frames were limited to $50 \mathrm{~ms}$ in order to support ASTERIA's $20 \mathrm{~Hz}$ fine pointing control loop (Pong 2018).

We began the data reduction process by reviewing every frame from the science observations. We manually flagged and discarded frames that were obtained during orbital sunrise or sunset. These frames appeared either at the beginning of an orbit or toward the end of the orbit, and displayed significant background contamination.

After discarding the sunrise/sunset frames, we proceeded to calibrate. We started with bias correction by subtracting the median of the bias column (from the calibration window) from all pixels in the respective column in the science frame. We then selected a few pixels (rows 0-15) in the neighborhood of the target that were devoid of any stellar flux to remove background and/or sky noise. We took the median of the pixels for each column, and subtracted the median value from every pixel in that column. Next, we subtracted the median of the bias rows column by column from every flat frame, and took the median of the reduced flat frame. Lastly, we performed a pixel-by-pixel division of the target star pixels with the reduced flat-frame pixel values. Note that the flat frame used to calibrate HD 219134 data was generated in space using stray light from the Sun to uniformly illuminate the detector as explained earlier (and see Knapp et al. 2020). A representative raw and calibrated image set is shown in Figure 4. The image of the target star is much cleaner than the raw image, although there is still some residual column-dependent noise. For more details on data reduction see Knapp et al. (2020) and Krishnamurthy (2020).

\subsubsection{ASTERIA Aperture Photometry and Detrending}

For aperture photometry, we began by calculating the target $x / y$ position by taking the flux-weighted moments for each pixel in the subarray window. We took the mean centroid position as the target star center, and performed photometric flux extraction using the CircularAperture routine from the photutils (Bradley et al. 2019) python package. We used fixed hard-edge apertures with radii ranging from 5 to 13 pixels. We determined the optimal aperture size by computing the photometric precision for each aperture size given by the rms of the light curve. We obtained an optimal aperture with a radius of 10 pixels based on maximization of the photometric precision (illustrated in Figure 4). We then proceeded to perform photometric flux extraction for all of the HD 219134 data.

For light-curve detrending, we used the Markov Chain Monte Carlo (MCMC) algorithm implementation from Gillon et al. (2012). Inputs to the MCMC were the HD 219134 photometric time series. We explored different functional forms of the baseline model for each light curve. These models could include a linear or quadratic trend with time, a second-order logarithmic ramp (Knutson et al. 2007; Demory et al. 2011) usually included for telescope settling with Spitzer and the Hubble Space Telescope (HST), a polynomial of the (1) centroid position and (2) onboard temperature measured at the lens housing, as well as linear combinations of the point-spread function (PSF) FWHM along the $x$ and $y$ axes. We employed the Bayesian information criterion (BIC; Schwarz 1978) to discriminate between the different baseline models. During our tests, we found that the baseline model resulting in the lower BIC (Schwarz 1978) was a combination of a second-order polynomial of (1) time, (2) $x / y$ centroid position, (3) lens 


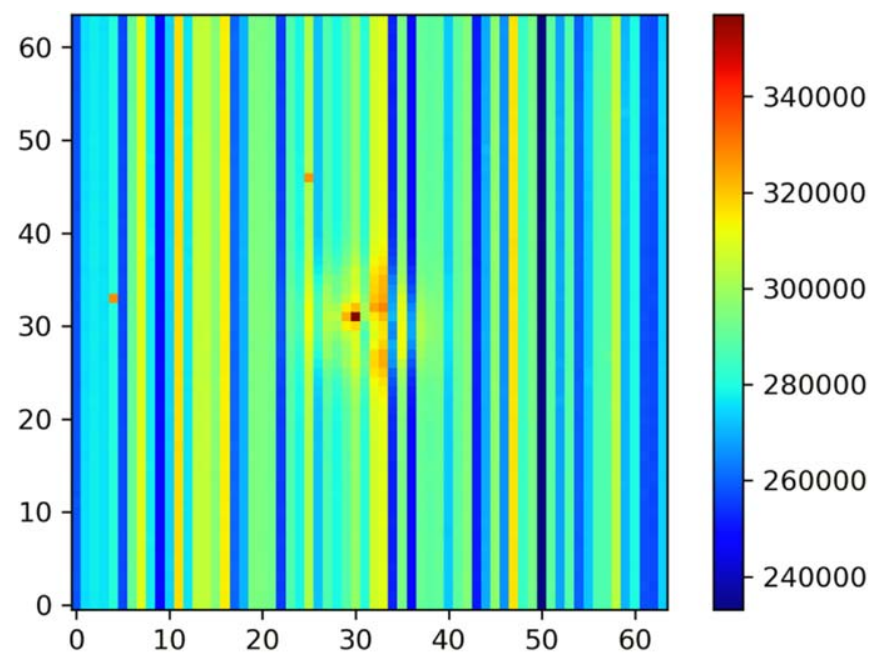

generate a $T_{0}$ span similar to the maximal gradient of the TTV pattern across the ASTERIA observations.

Our transit injection and recovery scheme was thus unusual in the sense that both the periods and transit midtimes were free to vary only within Gaussian priors defined by the transit ephemerides, testing the detection threshold for different radii of HD $219134 \mathrm{f}$ and $\mathrm{d}$.

For both planets $\mathrm{f}$ and $\mathrm{d}$ we performed injection and recovery tests for radius values ranging between 1.0 and $4.0 R_{\oplus}$, in steps of $0.25 R_{\oplus}$. ASTERIA observations were limited to 30 minute intervals of night out of a total 90 minute orbit, resulting in noncontinuous observations. The transit injection and recovery does not work as effectively in sparsely sampled data as compared to continuous data, as a large fraction of the transits could fall where data do not exist, hence reducing the detection significance. Because of the low on-target duty cycle, the detection capability for each injected transit is strongly dependent on ASTERIA's resulting window function. Slight changes to the planet's period and/or midtransit ephemerides could then affect the recovery efficiency for both signals. We required a signal-to-noise ratio of 5 .

\subsection{TESS Observations}

TESS observed HD 219134 in camera 3 during Sector 17, from 2019 October 7 to 2019 November 2 and in camera 4 during Sector 24. HD 2190134 has the TESS Input Catalog number TIC 283722336 (Stassun et al. 2018).

The $3 \sigma$ transit time window of HD $219134 \mathrm{f}$ falls within the TESS observations, based on the ephemeris from Gillon et al. (2017). In contrast, $3 \sigma$ transit window of HD $219134 \mathrm{~d}$ falls outside of the TESS observations.

\subsection{TESS Data Analysis}

TESS data calibration, reduction, detrending, and light-curve fitting is covered in a large number of papers (e.g., Huang et al. 2018; Vanderburg et al. 2019; Dragomir et al. 2019; Huang et al. 2020). Here we provide a summary of our process.

\subsubsection{TESS Data Reduction and Aperture Photometry}

We used the calibrated TESS two-minute cadence data produced by the Science Processing Operations Center (SPOC) pipeline based at NASA Ames Research Center (Jenkins 2015; Jenkins et al. 2016).

Because of its considerable brightness $\left(T_{\mathrm{mag}} \sim 4.6\right), \mathrm{HD} 219134$ saturated on the TESS CCDs. We chose to optimize the photometry based on our past experience working with TESS bright stars (Huang et al. 2018; Vanderburg et al. 2019). We reextracted the photometry using a series of irregular shaped apertures placed on the SPOC-calibrated target pixel stamp. We chose the aperture that minimized the scatter in the out-of-transit portion of the light curve (Figure 5). We next used the SPOC quality flags to eliminate low-quality data points.

For bright stars, the TESS systematics are mainly due to the pointing jitter of the spacecraft that occur on timescales shorter than the exposure time. We tried to remove such pointing systematics using the standard deviation of quaternion measurements within an exposure (Vanderburg et al. 2019), the first seven SPOC Presearch Data Conditioning (PDC) cotrending basis vectors $^{18}$, and also a trigonometric polynomial for longer-term planet sizes at the HD $219134 \mathrm{f}$ and d periods and varied the transit midtimes using a Gaussian distribution matching our calculated transit-timing variation (TTV) amplitudes (see Section 2.5). The period was also allowed to vary in order to

\footnotetext{
18 The cotrending basis vectors can be downloaded from the MAST CBV bulk download page.
} 


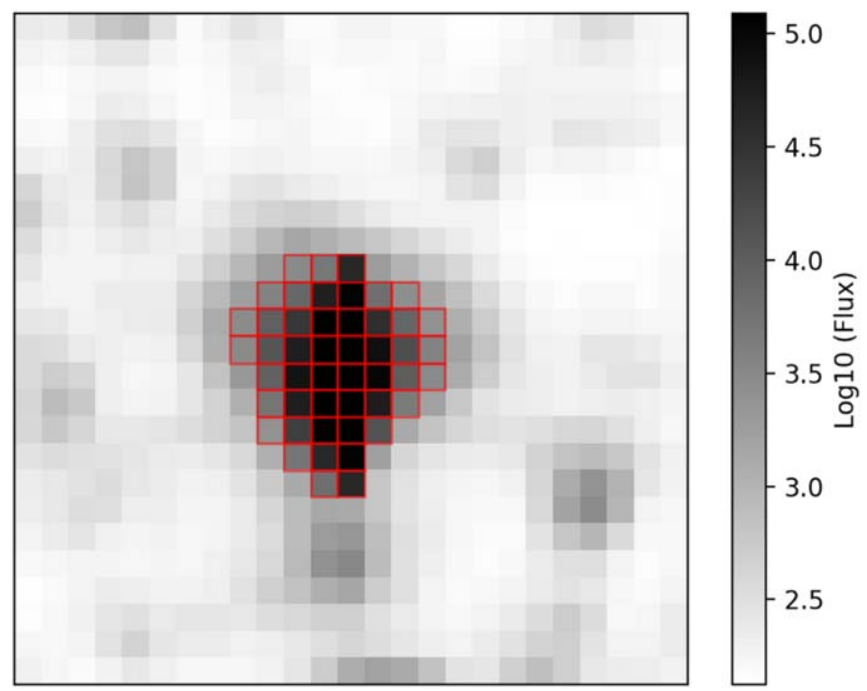

Figure 5. TESS custom aperture for HD 219134 on a log scale.

trends. We used a least-squares method to optimize the detrending coefficients by minimizing the out-of-transit scatter of the detrended light curve (for details on the the SPOC PDC see Smith et al. 2012 and Stumpe et al. 2012, 2014).

\subsubsection{TESS Transit Injection and Recovery Tests}

To search for transits in the TESS data we performed an injection and recovery of transits. We generated transits using BATMAN (Kreidberg 2015) and then injected the transits into the pre-detrended light curve. We investigated the detection rate as a function of planet radii using a grid of planet sizes. The radius grid was $0.5 \leqslant R_{p}\left(R_{\oplus}\right) \leqslant 1.75$ with uniform steps of $0.05 R_{\oplus}$, and a slightly larger grid using uniform steps of $0.15 R_{\oplus}$ for larger planetary radii $1.75<R_{p}\left(R_{\oplus}\right) \leqslant 4.0$.

For each planet size, we estimated the detection rate using a binomial distribution from 40 trials. Each trial consisted of injected planets at a given size, using an impact parameter drawn from a uniform distribution between 0 and 1 , a randomized $T_{0}$ with no other restrictions except that $T_{0}$ falls within the TESS Sector 17 and 24 data, and the period of the planet using the prior constraint from Gillon et al. (2017). Although the $T_{0}$ and small planet size search parameters are extraneous, we do this to be as exhaustive as possible with our transit search. We then followed the same detrending procedure described above (Section 2.4.1) and ran a box least-square (BLS) search with the period fixed at the bestfitted value from Gillon et al. (2017). For any detected signal, we required the BLS algorithm to recover an epoch within less than half the transit duration of the injected $T_{0}$, and the signal-to-noise ratio of the recovered transit to be larger than 7.3.

\subsection{Transit-timing Variation Methods}

We evaluate the range of transit times of planets $f$ and $d$, considering TTVs of mutual gravitational interactions of the four planets (b, c, f, and d). We used analytic formulae that are accurate to the first order in mass ratios and eccentricity, from Agol \& Deck (2016). We verified the validity of the transit times from our analytic approach by using an $\mathrm{N}$-body integrator REBOUND (Rein \& Liu 2012), but opted to use the faster analytic approach for our results for efficiency.

We ran a Monte Carlo analysis of the expected TTV amplitudes using a distribution of planetary parameters of the planets in the

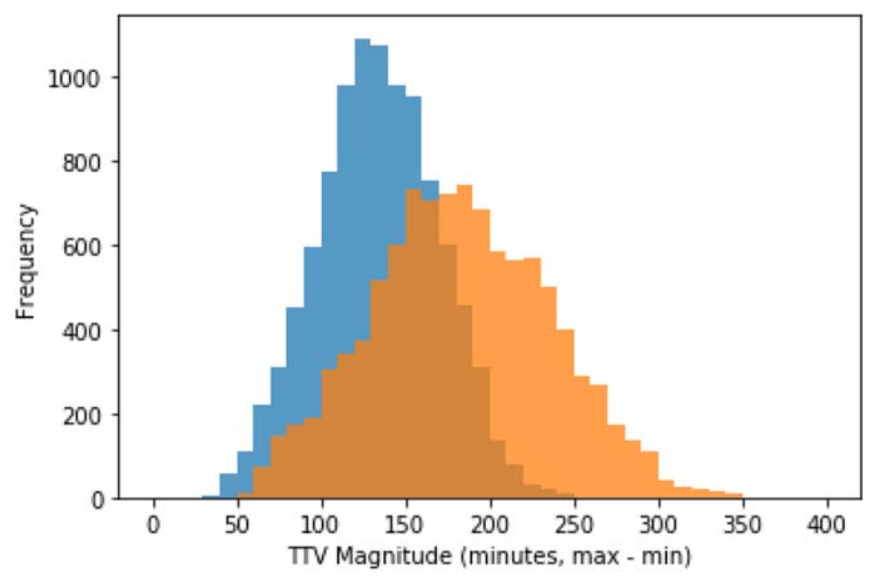

Figure 6. Transit-timing variation (TTV) magnitudes for HD 219134 d (blue) and $\mathrm{f}$ (orange).

HD 219134 system to analyze the maximum expected shift in observed time of transit due to TTVs. For this purpose, we used the median values and credible intervals from the global MCMC fit of results from Gillon et al. (2017) to reconstruct Gaussian posteriors for the orbital parameters of planets HD $219134 \mathrm{~b}, \mathrm{c}, \mathrm{f}$, and $d$. This combined fit used HARPS-N data and three Spitzer transits (two of planet $b$ and one of planet c) as input. We performed 10,000 draws from these distributions, preserving covariances, and simulated the expected TTV amplitudes for each draw (Figure 6). We used the resulting distributions of TTVs as inputs to the injection and recovery tests in the ASTERIA data, using different $T_{0}$ and period offsets at each trial. The conclusions from our TTV analysis are discussed in Section 3.3.

\section{Results and Discussion}

We do not find transit signals of HD $219134 \mathrm{f}$ or $\mathrm{d}$ in either the ASTERIA or TESS data.

\subsection{ASTERIA Results and Discussion}

The ASTERIA data are sensitive to planets as small as $3.7 R_{\oplus}$ for HD $219134 \mathrm{f}$ and $3.6 R_{\oplus}$ for $\mathrm{HD} 219134 \mathrm{~d}$ at the $5 \sigma$ threshhold. These results are based on our injection and recovery completeness tests (see Section 2.2.4). For comparison, known exoplanets with masses within 10\% that of HD 219134 d's radialvelocity measured minimum mass of $16.2 M_{\oplus}$, empirically fall into a radius range ${ }^{19}$ from 1.7 to $7.0 R_{\oplus}$. Uranus at $14.54 M_{\oplus}$ has a radius of $4.007 R_{\oplus}$ and Neptune at $17.15 M_{\oplus}$ has a radius of $3.883 R_{\oplus}$. For HD $219134 \mathrm{f}$ and d, ASTERIA therefore can rule out planets larger than the established limits, and those limits are somewhat smaller than Uranus and Neptune.

We are unable to detect transits $\mathrm{b}$ and $\mathrm{c}$ in our ASTERIA data. HD 219134 planets b and c are small, 1.6 and $1.5 R_{\oplus}$ respectively (Table 1), with shallow transits of $358 \pm 24$ and $315 \pm 19$ ppm respectively, from Spitzer data (Gillon et al. 2017). We ran the injection and recovery completeness test on the ASTERIA data for the radial-velocity ephemeris of HD $219134 \mathrm{~b}$ and found that the injected planet was retrieved systematically at a radius $>2.7 R_{\oplus}$, which is larger than the measured planet radius of $1.6 R_{\oplus}$. For planet HD $219134 \mathrm{c}$ the injected planet was recovered at a radius $>4.0 R_{\oplus}$, which is larger than the actual radius of $1.5 R_{\oplus}$.

\footnotetext{
${ }^{19}$ http://exoplanet.eu
} 

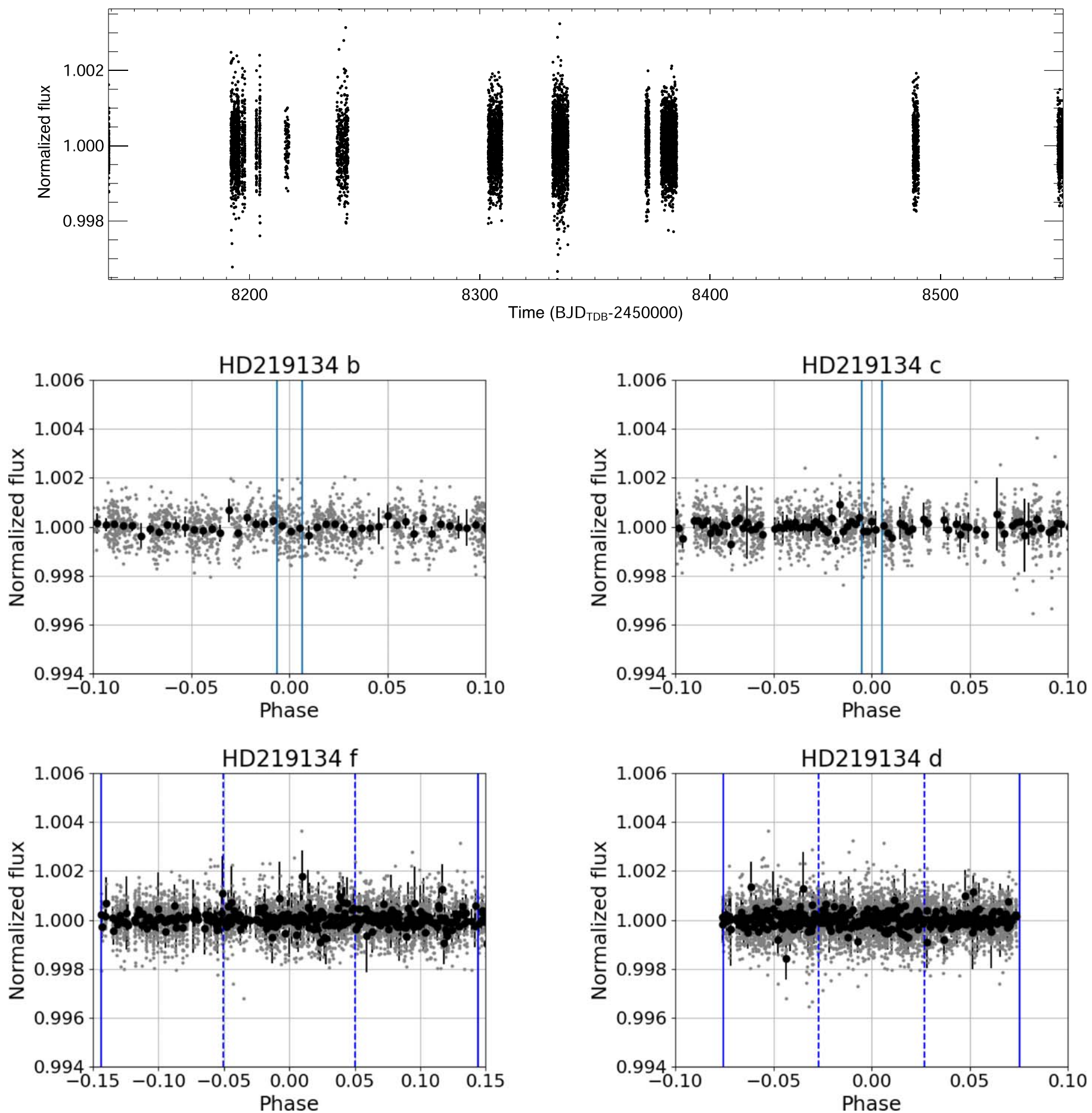

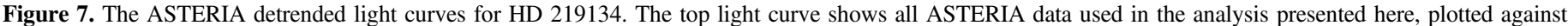

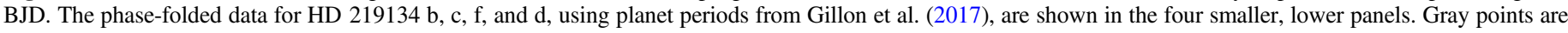

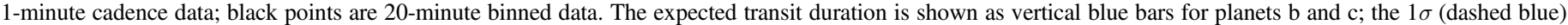
and $3 \sigma$ (solid blue) transit windows are shown for planets $\mathrm{f}$ and $\mathrm{d}$.

The limiting factor in our transit nondetections is ASTERIA's residual correlated noise. Indeed ASTERIA reaches a photometric precision typically within a factor of 2 to 3 of the photon noise due to the residual correlated noise which we are neither able to attribute nor remove. The photon noise for HD 219134 is $350 \mathrm{ppm}$ per minute, whereas our photometric precision for different observing nights ranges from $502 \mathrm{ppm}$ per minute (1.4 times the photon noise) to $1038 \mathrm{ppm}$ per minute (3.0 times the photon noise; Appendix). The overall ASTERIA HD 219134 data set has a median of 673 ppm per minute photometric precision. We show the ASTERIA HD 219134 detrended light curves as a time series and also phase-folded for each planet b, c, f, and d in Figure 7.

\subsection{TESS Results and Discussion}

The SPOC pipeline automatically detected the transits of HD 219134b and HD 219134c. The TESS Science Office designated the planets TOI 1469.01 and 1469.02 respectively. 

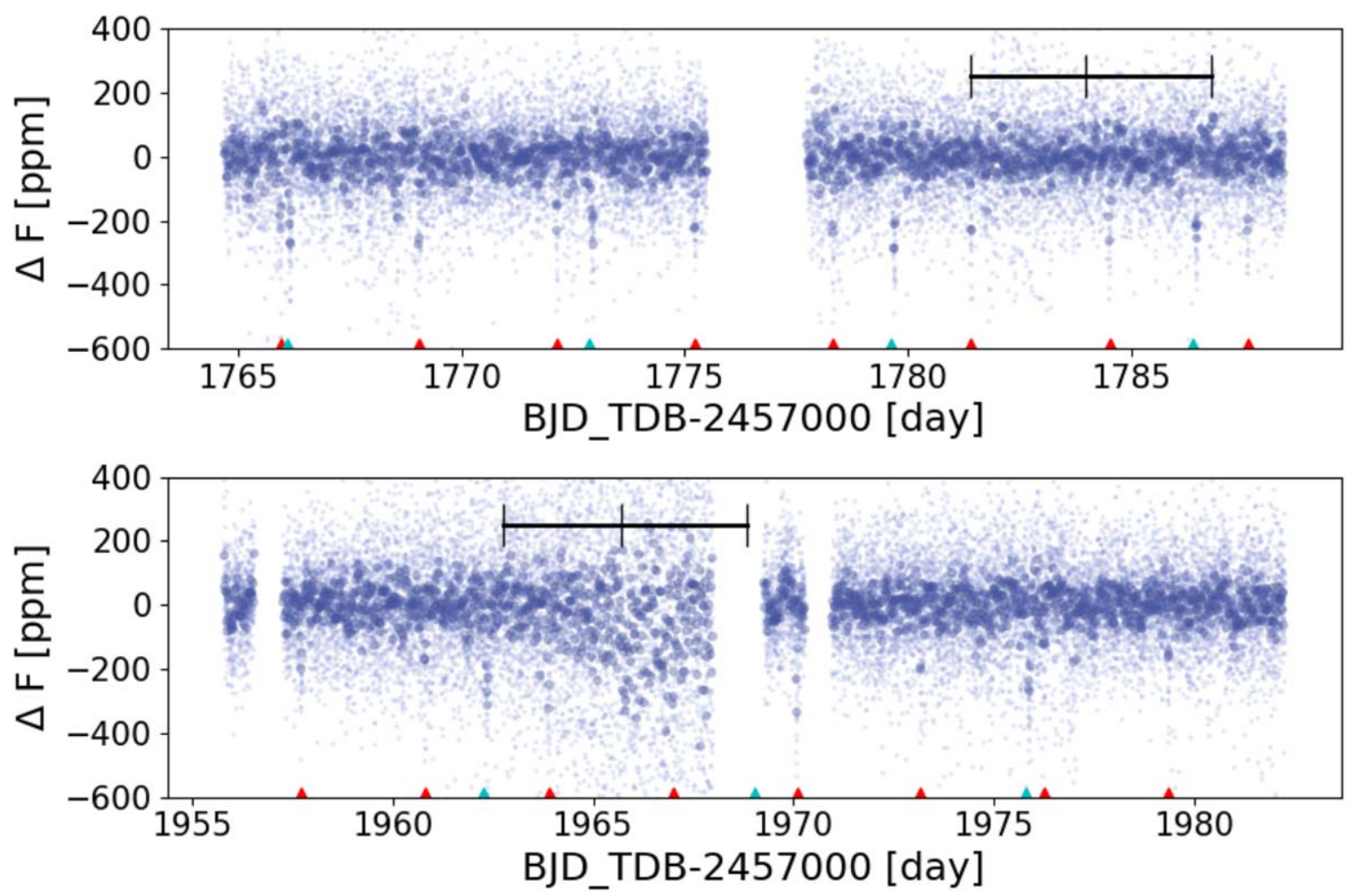

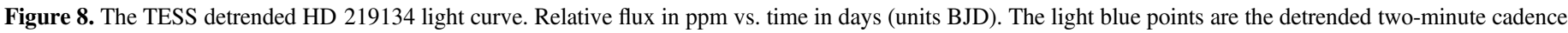

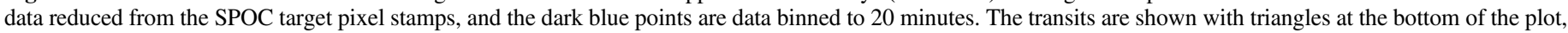

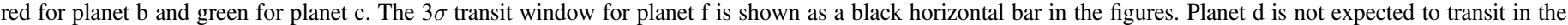

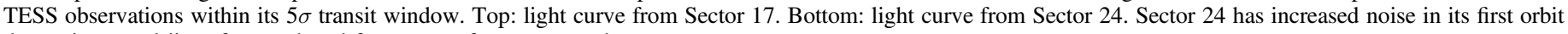
due to increased jitter from reduced frequency of momentum dumps.

The known transits of HD $219134 \mathrm{~b}$ and c are clearly apparent in our detrended TESS two-minute cadence data (Figure 8).

We derived updated ephemerides for planets HD $219134 \mathrm{~b}$ and $\mathrm{c}$ using the MCMC method as implemented by the allesfitter code (Günther \& Daylan 2020). In particular, we ran our MCMC routine with 100 walkers, 20000 steps, and a burn-in of 2000. For each free parameter, we ensured convergence by requiring that the chain lengths are at least 50 times the samplers autocorrelation time (Foreman-Mackey et al. 2013). The median and $1 \sigma$ uncertainties of the posterior distributions, including the updated orbital periods and midtransit times are presented in detail in Table A3, with a summary of the midtransit times and periods in Table 3 . We present the corresponding transit $\mathrm{b}$ and $\mathrm{c}$ fits in the Appendix.

Beyond the known transits of HD $219134 \mathrm{~b}$ and $\mathrm{c}$, no additional transits are detected in the SPOC light curves by the pipeline. We also find with our own detrended SPOC data that there are no obvious signs of any additional transit-like signals in the TESS Sectors 17 or 24 HD 219134 light curves.

Our TESS-data-derived radius lower limit for HD $219134 \mathrm{f}$ is $1 R_{\oplus}$, based on our injection and recovery tests (Section 2.4.2; Figure 9). This value is stringent enough to rule out transits.

Our derived lower limit of $1 R_{\oplus}$ is smaller than the $1.3 R_{\oplus}$ for a pure iron planet for HD 219134 f's radial-velocity minimum mass of $7 M_{\oplus}$ (e.g., Fortney et al. 2007; Seager et al. 2007; Zeng et al. 2016; Gillon et al. 2017). More specifically, we derive a lower limit of $0.925 R_{\oplus}$ and $1.25 R_{\oplus}$ for a $50 \%$ and $80 \%$ completeness in the recovery rate, respectively. With our derived lower radius limit smaller than any plausible planet, we thus rule out HD $219134 \mathrm{f}$ as a transiting planet.
Table 3

HD $219134 \mathrm{~b}$ and c Updated Midtransit Times $\left(T_{0}\right)$ and Planet Orbital Periods $(P)$ from Our Detrended TESS SPOC Two-minute Cadence Data

\begin{tabular}{lcc}
\hline \hline Planet Parameter & Planet b & Planet c \\
\hline$T_{0}$ (BJD) & $2458765.95501 \pm 0.00047$ & $2458766.16927_{-0.00066}^{+0.00069}$ \\
$P$ (days) & $3.092920 \pm 0.000011$ & $6.765149_{-0.000036}^{+0.00033}$ \\
\hline
\end{tabular}

Note. The values are median values with $1 \sigma$ uncertainties.

With a robust nondetection, we can state that, if real, that inclination of HD $219134 \mathrm{f}$ is less than $88^{\circ} .6$ (assuming we cannot detect a grazing transit).

TESS provides no constraint on HD $219134 \mathrm{~d}$ which did not pass through inferior conjunction during the TESS observations.

\subsection{Transit-timing Variation Results and Discussion}

From our TTV analysis (Section 2.5) we find no evidence that TTVs would have prevented either planet $\mathrm{f}$ or $\mathrm{d}$ from being detectable with the current recovery algorithms.

The TTV amplitude of HD $219134 \mathrm{f}$ (Figure 6) could have been larger than the expected transit duration. However, since planet $f$ should have transited once during the TESS data with a depth of about $238 \mathrm{ppm}$, it should have been detectable regardless of TTVs. Specifically we found that given the samples of orbital parameters, the distribution of TTVs ranges from 50 minutes to 350 minutes, larger than the expected transit duration of $\lesssim 230$ minutes (Table 1 ).

The potential TTV amplitude of HD $219134 \mathrm{~d}$ (Figure 6) is smaller than the expected transit duration. More specifically we 


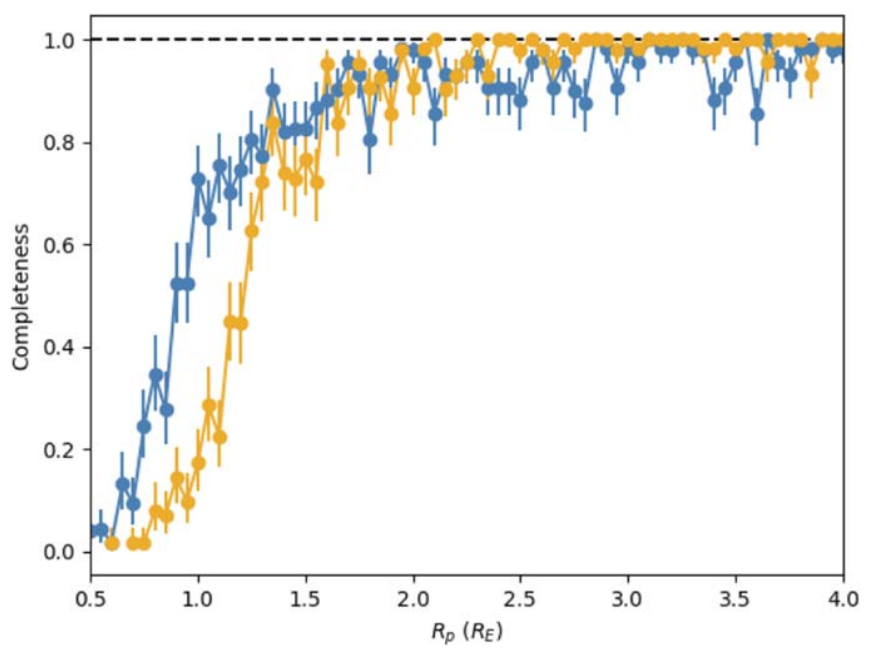

Figure 9. Completeness in the TESS short-cadence light curves for HD 219134 planets $\mathrm{f}$ and $\mathrm{d}$ for different size planets. The $y$-axis represents the probability of detecting the planets of a given size if the transit occurred any time within the TESS observation data. The blue curve is for planet $\mathrm{f}$ and the orange curve is for planet $\mathrm{d}$. The $x$-axis is planet radius in Earth radii. The error bars are estimated using the binomial distribution from 40 random draws of impact parameter and transit epochs. We can confidently rule out planet $\mathrm{f}$ transits as long as planet $\mathrm{f}$ is larger than $1 R_{\oplus}$, which it is expected to be based on planet models for the maximum density of the minimum mass of planet $\mathrm{f}$.

find that given the samples of orbital parameters, the distribution of TTVs ranges from 50 minutes to 250 minutes, less than the expected transit duration of $\lesssim 340$ minutes (Table 1). Since the vast majority of TTV amplitudes are within the expected transit duration, TTVs most likely did not have an effect on the planet being observed.

A limitation to our TTV approach is that we used results from a Keplerian fit to infer TTV amplitudes that result from non-Keplerian dynamics. Another limitation is that both injection and recovery algorithms used linear ephemeris parameterization. One could push the TTV analysis to further accuracy by performing a full $N$-body photodynamical fit of the radial-velocity data and the photometry, including a nonKeplerian fit. Such an approach, however, is not warranted in the present paper because the expected transit depths of planets $\mathrm{f}$ and $\mathrm{d}$ are below the sensitivity threshold of ASTERIA.

\section{Summary}

We revisited the bright nearby star HD 219134 with TESS and ASTERIA. With the TESS data, we provided updated transit times and periods for the known transiting planets HD $219134 \mathrm{~b}$ and $\mathrm{c}$. The planet $\mathrm{f}$ transit window fell within TESS Sector 17 and 24 data; we completely rule out transits for planet $f$ based on the nondetection of planets smaller than a pure iron planet corresponding to the measured minimum mass of planet $f$.

We used the ASTERIA data to place upper limits on HD 219134 planet d transits (for which the transit window did not fall within the TESS data). ASTERIA was sensitive to planet $\mathrm{d}$ sizes down to a radius of $3.6 R_{\oplus}$. There is still room for planet $d$ to show transits, because planets within $10 \%$ of the mass of planet d's minimum mass have an empirical radius range from 1.7 to $7.0 R_{\oplus}$.

ASTERIA was a technology demonstration mission that successfully achieved the pointing precision and thermal control necessary for precise photometry. Without design and calibration data for highly precise photometry, however, ASTERIA's residual uncorrelated noise ultimately limited the photometric precision and scientific capabilities. Nonetheless, ASTERIA technology demonstration success shows what is possible within a small satellite (specifically a CubeSat) platform.

ASTERIA data for HD 219134 is archived at https:// exoplanetarchive.ipac.caltech.edu/docs/ASTERIAMission.html and TESS data for HD 219134 is archived at MAST (https:// archive.stsci.edu/).

We acknowledge contributions from the extended team that supported ASTERIA development, integration and test, and operations, including Len Day, Maria de Soria Santacruz-Pich, Carl Felten, Janan Ferdosi, Kristine Fong, Harrison Herzog, Jim Hofman, David Kessler, Roger Klemm, Jules Lee, Jason Munger, Lori Moore, Esha Murty, Chris Shelton, David Sternberg, Rob Sweet, Kerry Wahl, Jacqueline Weiler, Thomas Werne, Shannon Zareh, and Ansel Rothstein-Dowden. We also recognize the JPL line organization and technical mentors for the expertise they provided throughout the project.

We thank the JPL program management, especially Sarah Gavit and Pat Beauchamp, who oversaw ASTERIA within the Engineering and Science Directorate at JPL. We also thank Daniel Coulter and Leslie Livesay for their support. The research was carried out in part at the Jet Propulsion Laboratory, California Institute of Technology, under a contract with the National Aeronautics and Space Administration. Government sponsorship acknowledged.

We would also like to thank the DSS-17 ground station team at Morehead State University (MSU) in Kentucky. We acknowledge the outstanding efforts of the student operators, technical staff, and program management at Morehead State University, including Chloe Hart, Sarah Wilczewski, Alex Roberts, Maria Lemaster, Lacy Wallace, Rebecca Mikula, Bob Kroll, Michael Combs, and Benjamin Malphrus.

Funding for the TESS mission is provided by NASA's Science Mission directorate. This paper includes data collected by the TESS mission, which are publicly available from the Mikulski Archive for Space Telescopes (MAST). Resources supporting this work were provided by the NASA High-End Computing (HEC) Program through the NASA Advanced Supercomputing (NAS) Division at Ames Research Center for the production of the SPOC data products. J.C.B. has been supported by the Heising-Simons 51 Pegasi $b$ postdoctoral fellowship.

We acknowledge the Massachusett Tribe, the tribe of indigenous peoples from whom the Colony, Province, and Commonwealth of Massachusetts have taken their name. We would like to pay our respects to the ancestral bloodline of the Massachusett tribe and their descendants who still inhabit historical Massachusett tribe territories to this day.

Facilities: TESS, ASTERIA, MAST.

Software: SPOC R4.0, QLP, Numpy (van der Walt et al. 2011), Scipy (Virtanen et al. 2020), Astropy (Astropy Collaboration et al. 2013, 2018), allesfitter (Günther \& Daylan 2020), photutils (Bradley et al. 2019).

\section{Appendix}

The Appendix contains figures and tables to further describe HD 219134 properties (Table A1), ASTERIA data (Figure A1, Table A2), TESS data (Figure A2), and TESS data-derived HD 219134 planet and system parameters (Table A3). 

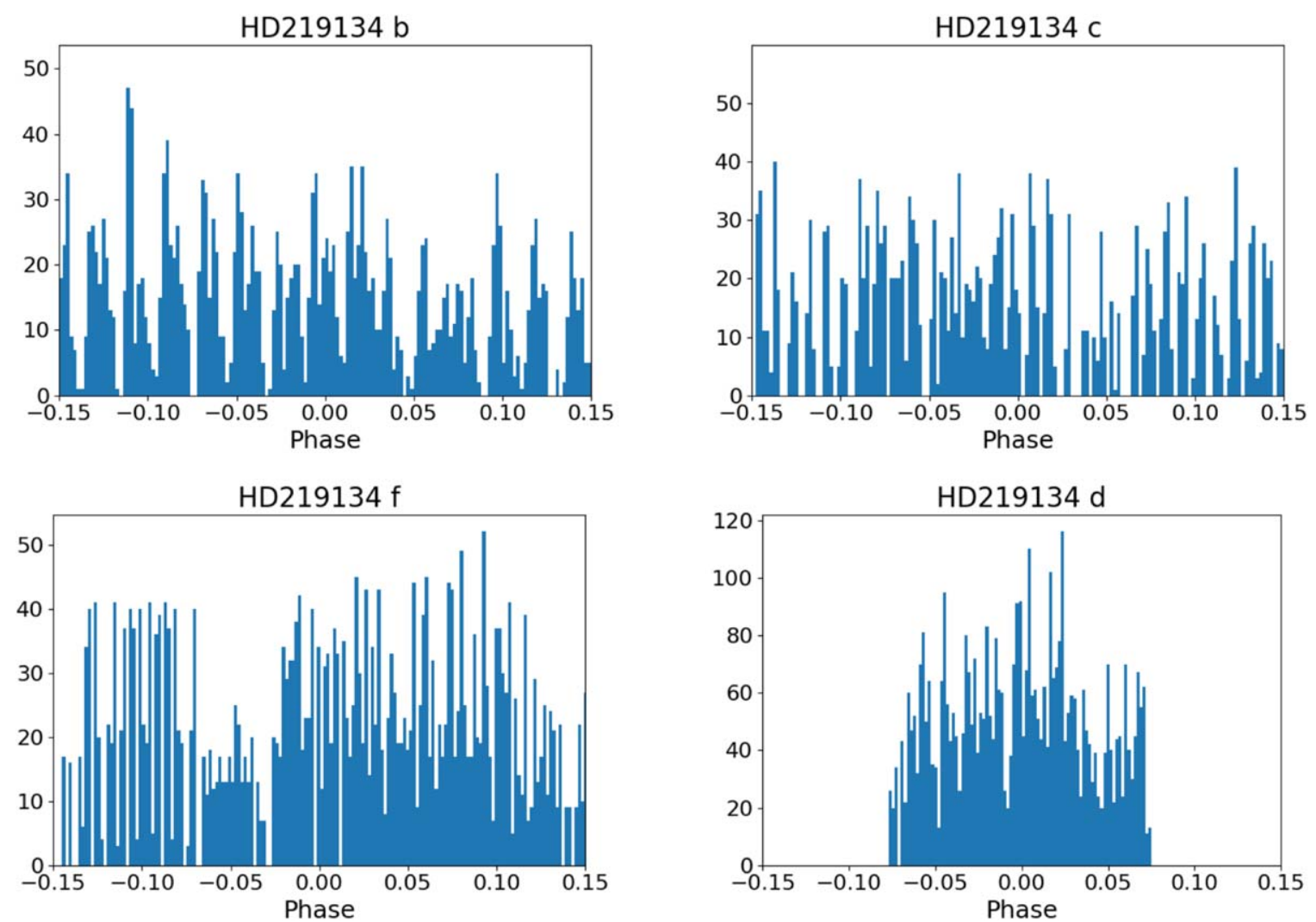

Figure A1. ASTERIA data density per planet phase (500 bins spaced uniformly in planet phase). The counts on the vertical axes are number of 1-minute cadence data points per bin.

Table A1

Stellar Properties of HD 219134

\begin{tabular}{lcc}
\hline \hline Property & Value & Source \\
\hline R.A. (J2015.5; hh:mm:ss) & $23: 13: 16.780$ & 1 \\
decl.. (J2015.5; dd:mm:ss) & $+57: 10: 10.05$ & 1 \\
$\mu_{\alpha}$ (mas yr $^{-1}$ ) & $2074.517 \pm 0.137$ & 1 \\
$\mu_{\delta}\left(\right.$ mas yr $\left.^{-1}\right)$ & $294.936 \pm 0.123$ & 1 \\
Parallax (mas) & $153.0808 \pm 0.0895$ & 1 \\
Distance (pc) & $6.5313 \pm 0.0039$ & 2 \\
\hline
\end{tabular}

Photometric Magnitudes

\begin{tabular}{lcc}
\hline$V$ (mag) & $5.570 \pm 0.009$ & 3 \\
$B$ (mag) & $6.560 \pm 0.007$ & 3 \\
TESS (mag) & $4.628 \pm 0.007$ & 2 \\
Gaia (mag) & $5.2079 \pm 0.0016$ & 1 \\
$J$ (mag) & 3.86 & 3.40 \\
$H$ (mag) & 3.25 \\
$K_{s}$ (mag) & 4 \\
\hline
\end{tabular}

\begin{tabular}{lcc}
\hline & Spectroscopic Properties \\
\hline$M_{\star}\left(M_{\odot}\right)$ & $0.81 \pm 0.03$ & 5 \\
$R_{\star}\left(R_{\odot}\right)$ & $0.778 \pm 0.005$ & 7 \\
$T_{\text {eff }}(\mathrm{K})$ & $4700 \pm 20$ & 7 \\
$\log g(\mathrm{dex})$ & $4.567 \pm 0.018$ & 5 \\
{$[\mathrm{Fe} / \mathrm{H}](\mathrm{dex})$} & $0.11 \pm 0.04$ & 6 \\
$\rho_{\star}\left(\rho_{\odot}\right)$ & $1.729 \pm 0.073$ & 5 \\
$L_{\star}\left(L_{\odot}\right)$ & $0.2646 \pm 0.0050$ & 5 \\
Age $(\mathrm{Gyr})$ & $11.0 \pm 2.2$ & 5 \\
\hline
\end{tabular}

Note. (1) Gaia DR2 (Gaia Collaboration et al. 2016). (2) TESS Input Catalog Version 8 (TICv8), Stassun et al. (2018). (3) Oja (1993). (4) Ducati (2002). (5) Gillon et al. (2017). (6) Motalebi et al. (2015). (7) Boyajian et al. (2012). 
Table A2

ASTERIA HD 219134 Observations Used in the Light Curve Shown in Figure 7

\begin{tabular}{|c|c|c|c|c|c|c|}
\hline $\begin{array}{l}\text { Observation } \\
\text { Name }\end{array}$ & $\begin{array}{c}\text { First Frame } \\
\text { [UTC] }\end{array}$ & $\begin{array}{l}\text { Last Frame } \\
\text { [UTC] }\end{array}$ & $\begin{array}{l}\text { Number of } \\
\text { Orbits }\end{array}$ & $\begin{array}{l}\text { Number of 1-minute } \\
\text { Integrations }\end{array}$ & Transit Window & $\begin{array}{c}\mathrm{rms} \\
{[\mathrm{ppm} / \mathrm{minute}]}\end{array}$ \\
\hline $\begin{array}{l}\text { Tech Demo } 22 \\
(\operatorname{td} 22)\end{array}$ & 2018-01-20 06:26:38.6 & 2018-01-20 11:23:28.0 & 4 & 79 & None & 592 \\
\hline $\begin{array}{l}\text { Science Observation } 08 \\
\text { (so08) }\end{array}$ & 2018-03-14 10:18:11.4 & 2018-03-15 01:55:17.1 & 11 & 164 & f, d & 882 \\
\hline $\begin{array}{l}\text { Science Observation } 09 \\
\text { (so09) }\end{array}$ & 2018-03-15 09:22:26.2 & 2018-03-16 02:33:57.8 & 9 & 154 & $f, d$ & 797 \\
\hline $\begin{array}{l}\text { Science Observation } 10 \\
\text { (so10) }\end{array}$ & $\begin{array}{l}\text { 2018-03-16 14:53:01.3 } \\
\text { 2018-03-16 19:15:16.7 }\end{array}$ & $\begin{array}{l}2018-03-18 \text { 05:27:40.3 } \\
2018-03-18 \text { 05:25:40.3 }\end{array}$ & $\begin{array}{r}5 \\
19\end{array}$ & 457 & f, d & $\begin{array}{l}701 \\
747\end{array}$ \\
\hline $\begin{array}{l}\text { Science Observation } 11 \\
\text { (so11) }\end{array}$ & 2018-03-19 07:17:15.5 & 2018-03-19 13:49:39.3 & & 95 & $b, d$ & 1023 \\
\hline $\begin{array}{l}\text { Science Observation } 12 \\
\text { (so12) }\end{array}$ & 2018-03-20 07:54:53.9 & 2018-03-20 11:23:33.7 & 3 & 70 & $\mathrm{c}, \mathrm{d}$ & 851 \\
\hline $\begin{array}{l}\text { Science Observation } 14 \\
\text { (so14) }\end{array}$ & 2018-03-25 09:49:25.5 & 2018-03-25 14:49:59.3 & 4 & 96 & $\mathrm{~b}$ & 695 \\
\hline $\begin{array}{l}\text { Science Observation } 15 \\
\text { (so15) }\end{array}$ & 2018-03-26 21:11:51.1 & 2018-03-27 02:12:17.3 & 4 & 96 & $\mathrm{c}$ & 1038 \\
\hline $\begin{array}{l}\text { Science Observation } 18 \\
\text { (so18) }\end{array}$ & 2018-04-06 23:49:45.4 & 2018-04-08 15:57:33.8 & 21 & 101 & $\mathrm{f}$ & 601 \\
\hline $\begin{array}{l}\text { Science Observation } 21 \\
\text { (so21) }\end{array}$ & $\begin{array}{l}2018-04-29 \text { 10:05:43.2 } \\
2018-04-29 \text { 20:52:26.2 } \\
\text { 2018-05-01 03:40:17.5 }\end{array}$ & $\begin{array}{ll}2018-04-29 & 14: 48: 51.3 \\
2018-04-30 & 21: 37: 43.6 \\
2018-05-01 & 16: 06: 28.9\end{array}$ & $\begin{array}{r}3 \\
11 \\
7\end{array}$ & 177 & $\mathrm{~b}, \mathrm{c}, \mathrm{f}, \mathrm{d}$ & $\begin{array}{l}624 \\
673 \\
650\end{array}$ \\
\hline $\begin{array}{l}\text { Science Observation } 22 \\
\text { (so22) }\end{array}$ & $\begin{array}{l}\text { 2018-05-01 22:09:01.0 } \\
\text { 2018-05-02 10:28:05.4 }\end{array}$ & $\begin{array}{l}\text { 2018-05-02 04:25:39.3 } \\
\text { 2018-05-04 07:16:21.3 }\end{array}$ & $\begin{array}{r}3 \\
25\end{array}$ & 264 & $f, d$ & $\begin{array}{l}585 \\
875\end{array}$ \\
\hline $\begin{array}{l}\text { Science Observation } 37 \\
\text { (so37) }\end{array}$ & $\begin{array}{l}\text { 2018-07-04 09:05:52.7 } \\
\text { 2018-07-05 20:28:50.8 }\end{array}$ & $\begin{array}{l}\text { 2018-07-05 16:07:55.4 } \\
\text { 2018-07-06 12:08:51.8 }\end{array}$ & $\begin{array}{r}18 \\
9\end{array}$ & 534 & $\mathrm{~b}, \mathrm{f}$ & $\begin{array}{l}695 \\
641\end{array}$ \\
\hline $\begin{array}{l}\text { Science Observation } 38 \\
\text { (so38) }\end{array}$ & $\begin{array}{l}2018-07-06 \quad 21: 05: 44.6 \\
2018-07-07 \text { 12:28:46.6 } \\
2018-07-08 \text { 14:37:54.9 }\end{array}$ & $\begin{array}{l}\text { 2018-07-07 06:35:35.0 } \\
2018-07-08 \text { 10:17:01.7 } \\
\text { 2018-07-09 } 03: 12: 51.3\end{array}$ & $\begin{array}{r}6 \\
12 \\
8\end{array}$ & 732 & $\mathrm{~b}, \mathrm{f}$ & $\begin{array}{l}617 \\
595 \\
645\end{array}$ \\
\hline $\begin{array}{l}\text { Science Observation } 39 \\
\text { (so39) }\end{array}$ & 2018-08-01 03:35:48.5 & 2018-08-02 18:23:49.6 & 19 & 309 & $\mathrm{c}, \mathrm{f}, \mathrm{d}$ & 720 \\
\hline $\begin{array}{l}\text { Science Observation } 40 \\
\text { (so40) }\end{array}$ & $\begin{array}{l}2018-08-03 \text { 03:15:31.9 } \\
2018-08-03 \text { 17:04:04.7 } \\
\text { 2018-08-04 08:27:57.1 } \\
2018-08-05 \text { 07:31:15.1 }\end{array}$ & $\begin{array}{l}2018-08-03 \quad 14: 20: 30.9 \\
2018-08-04 \quad 05: 43: 21.2 \\
2018-08-05 \quad 04: 45: 39.2 \\
2018-08-06 \quad 13: 03: 45.5\end{array}$ & $\begin{array}{r}7 \\
9 \\
12 \\
19\end{array}$ & 1061 & $\mathrm{~b}, \mathrm{~d}$ & $\begin{array}{l}986 \\
682 \\
710 \\
715\end{array}$ \\
\hline $\begin{array}{l}\text { Science Observation } 41 \\
\text { (so41) }\end{array}$ & 2018-08-07 01:03:01.7 & 2018-08-07 19:48:34.3 & 12 & 271 & d & 751 \\
\hline $\begin{array}{l}\text { Science Observation } 43 \\
\text { (so43) }\end{array}$ & 2018-09-10 14:10:58.7 & 2018-09-12 00:23:13.8 & 21 & 496 & $\mathrm{~b}, \mathrm{f}$ & 645 \\
\hline $\begin{array}{l}\text { Science Observation } 45 \\
\text { (so45) }\end{array}$ & 2018-09-17 07:43:57.2 & 2018-09-18 01:01:37.9 & 10 & 243 & $b, d$ & 575 \\
\hline $\begin{array}{l}\text { Science Observation } 46 \\
\text { (so46) }\end{array}$ & 2018-09-18 09:51:37.5 & 2018-09-19 21:37:50.1 & 19 & 474 & $\mathrm{~d}$ & 613 \\
\hline $\begin{array}{l}\text { Science Observation } 47 \\
\text { (so47) }\end{array}$ & 2018-09-20 08:00:54.7 & 2018-09-20 23:46:46.1 & 9 & 240 & $b, d$ & 640 \\
\hline $\begin{array}{l}\text { Science Observation } 48 \\
\text { (so48) }\end{array}$ & $\begin{array}{l}2018-09-21 \mathrm{~T} 05: 32: 52.0 \\
2018-09-22 \mathrm{~T} 19: 59: 26.0\end{array}$ & $\begin{array}{l}2018-09-22 \quad 15: 45: 40.3 \\
2018-09-24 \text { 00:02:48.8 }\end{array}$ & $\begin{array}{l}21 \\
15\end{array}$ & 851 & $b, d$ & $\begin{array}{l}622 \\
589\end{array}$ \\
\hline $\begin{array}{l}\text { Science Observation } 50 \\
\text { (so50) }\end{array}$ & 2019-01-04 15:45:39.4 & 2019-01-07 00:42:54.7 & 31 & 472 & $\mathrm{c}, \mathrm{f}$ & 701 \\
\hline
\end{tabular}


Table A2

(Continued)

\begin{tabular}{lccccr}
\hline \hline $\begin{array}{l}\text { Observation } \\
\text { Name }\end{array}$ & $\begin{array}{c}\text { First Frame } \\
{[\mathrm{UTC}]}\end{array}$ & $\begin{array}{c}\text { Last Frame } \\
{[\mathrm{UTC}]}\end{array}$ & $\begin{array}{c}\text { Number of } \\
\text { Orbits }\end{array}$ & $\begin{array}{c}\text { Number of 1-minute } \\
\text { Integrations }\end{array}$ & $\begin{array}{c}\text { Transit Window } \\
\text { [ppm/minute] }\end{array}$ \\
\hline Science Observation 64 & $2019-03-08$ 13:04:22.6 & $2019-03-0919: 59: 47.7$ & 15 & 541 & None \\
(so64) & $2019-03-1001: 50: 02.4$ & $2019-03-1102: 36: 50.7$ & 10 & 577 & 502 \\
\hline
\end{tabular}

Note. Observations designated "Tech Demo" or "td" took place during the initial phase of the mission when observations were focused on addressing ASTERIA's technology demonstration goals. After the technology demonstration objectives were completed, the naming scheme changed to "Science Observation" or "so" to indicate the change in focus from technology demonstration to collection of science data.
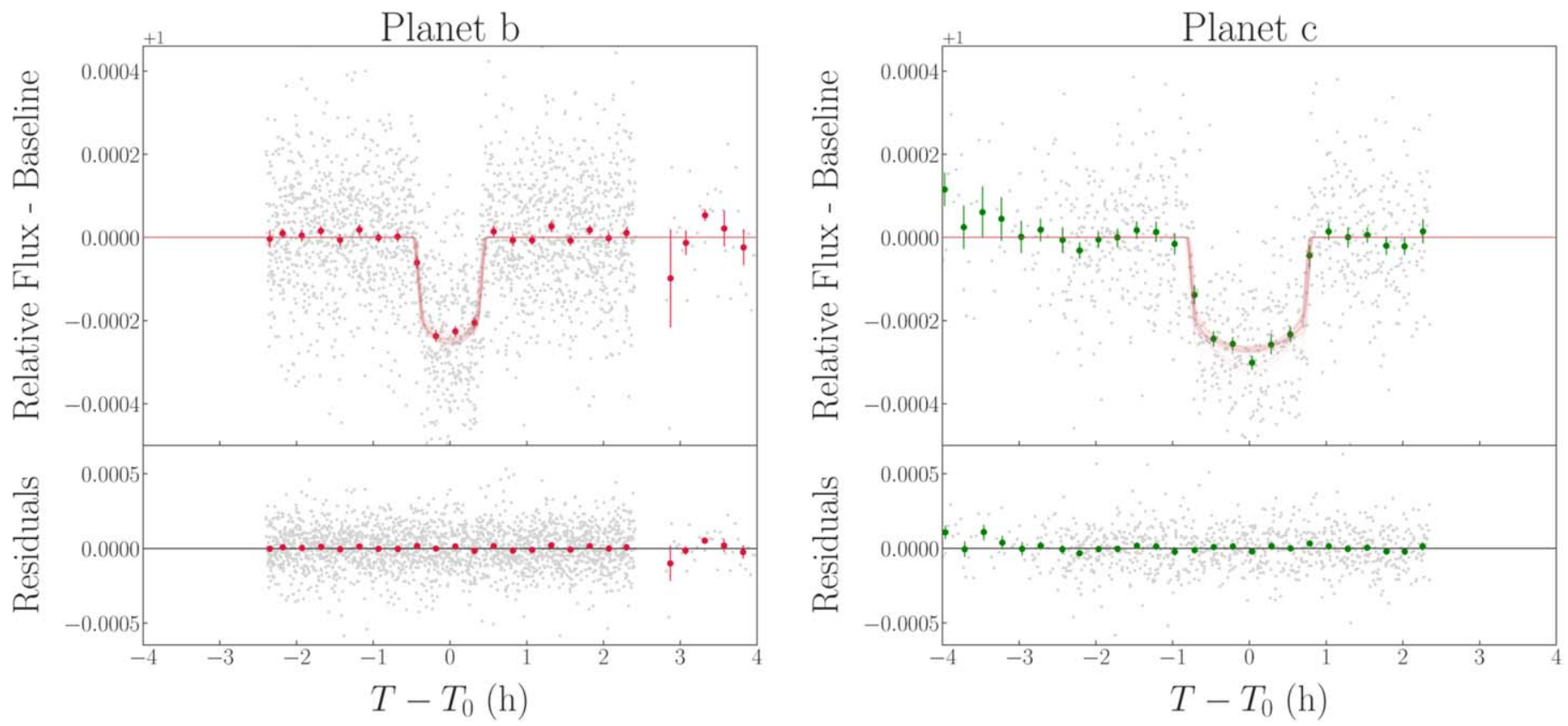

Figure A2. The TESS phase-folded light curves over the allesfitter best-fit orbital periods and midtransit times. (See Table A3). The gray points are the TESS unbinnned two-minute cadence data, the colored dots are the data binned over 15 minutes, and the red lines are 20 posterior models drawn randomly from the allesfitter posterior samples. The light-curve residuals are shown in the bottom panel. 
Table A3

Median Values and $1 \sigma$ Uncertainties for the HD 219134b and HD 219134c Planets. The Planet Radii and Semimajor Axes Are Less Precise Than in Gillon et al. (2017)

\begin{tabular}{|c|c|c|}
\hline Parameter & HD 219134b & HD $219134 \mathrm{c}$ \\
\hline Midtransit Time, $T_{0 ; \mathrm{p}}$ (BJD days) & $2458765.95501 \pm 0.00047$ & $2458766.16927_{-0.00066}^{+0.00069}$ \\
\hline Orbital Period, $P_{\mathrm{p}}$ (days) & $3.092920 \pm 0.000011$ & $6.765149_{-0.000036}^{+0.000033}$ \\
\hline Radius Ratio, $R_{\mathrm{p}} / R_{\star}$ & $0.01806_{-0.00091}^{+0.0011}$ & $0.01776_{-0.00070}^{+0.00086}$ \\
\hline Sum of Radii over Semimajor axis, $\left(R_{\star}+R_{\mathrm{p}}\right) / a_{\mathrm{p}}$ & $0.0954_{-0.0013}^{+0.0014}$ & $0.05647_{-0.00079}^{+0.00083}$ \\
\hline Cosine of Orbital Inclination, $\cos i_{\mathrm{p}}$ & $0.0870 \pm 0.0016$ & $0.04703 \pm 0.0010$ \\
\hline Transit Depth, $\delta_{\text {dil; } ; \text {;Ess }}(\mathrm{ppt})$ & $0.0002488_{-0.000010}^{+0.000009}$ & $0.000280_{-0.000013}^{+0.000012}$ \\
\hline Planet Radius, $R_{\mathrm{p}}\left(R_{\oplus}\right)$ & $1.533_{-0.078}^{+0.097}$ & $1.507_{-0.060}^{+0.074}$ \\
\hline Semimajor Axis, $a_{\mathrm{p}}(\mathrm{au})$ & $0.03863 \pm 0.00062$ & $0.0652 \pm 0.0010$ \\
\hline Orbital Inclination, $i_{\mathrm{p}}(\mathrm{deg})$ & $85.008 \pm 0.091$ & $87.304 \pm 0.059$ \\
\hline Impact Parameter, $b_{\text {tra; }}$ & $0.9293_{-0.0051}^{+0.0045}$ & $0.8479_{-0.0084}^{+0.0079}$ \\
\hline Total Transit Duration, $T_{\text {tot;p }}(\mathrm{h})$ & $0.925_{-0.020}^{+0.022}$ & $1.617_{-0.027}^{+0.029}$ \\
\hline Full Transit Duration, $T_{\text {full;p }}(\mathrm{h})$ & $0.704_{-0.027}^{+0.029}$ & $1.423_{-0.031}^{+0.033}$ \\
\hline Equilibrium Temperature, $T_{\text {eq;p }}(\mathrm{K})$ & $930.1 \pm 7.6$ & $715.9 \pm 5.8$ \\
\hline
\end{tabular}

System Parameters

Limb-darkening Coefficient $1, u_{1 ; \text { TESS }}$

Limb-darkening Coefficient 2, $u_{2 ; \text { TESS }}$

Flux Error, $\log \sigma_{\text {TESS }}$ (logrel. flux.)

GP Characteristic Amplitude, gp: $\log \sigma$ (TESS)

GP Timescale, gp: $\log \rho$ (TESS)

$$
\begin{gathered}
0.66 \pm 0.44 \\
-0.08_{-0.38}^{+0.44} \\
-8.9024 \pm 0.0040 \\
-10.472 \pm 0.056 \\
-2.11 \pm 0.21
\end{gathered}
$$

\section{ORCID iDs}

Sara Seager (i) https://orcid.org/0000-0002-6892-6948

Mary Knapp (iD https://orcid.org/0000-0002-5318-7660

Brice-Olivier Demory (iD https://orcid.org/0000-0002-9355-5165

Akshata Krishnamurthy (iD https://orcid.org/0000-0002-

8781-2743

Chelsea X. Huang (iD https://orcid.org/0000-0003-0918-7484

Mariona Badenas Agusti (i) https://orcid.org/0000-0003-

4903-567X

Avi Shporer (1D https://orcid.org/0000-0002-1836-3120

Drew Weisserman (1D https://orcid.org/0000-0002-7992-469X

Juliette Becker (i) https://orcid.org/0000-0002-7733-4522

Andrew Vanderburg (1) https://orcid.org/0000-0001-7246-5438

Matthew Smith (10 https://orcid.org/0000-0003-0103-8820

Vanessa P. Bailey (iD https://orcid.org/0000-0002-5407-2806

George Ricker (iD https://orcid.org/0000-0003-2058-6662

Roland Vanderspek (iD https://orcid.org/0000-0001-6763-6562

David W. Latham (iD https://orcid.org/0000-0001-9911-7388

Jon M. Jenkins (iD https://orcid.org/0000-0002-4715-9460

Joshua N. Winn (i) https://orcid.org/0000-0002-4265-047X

Douglas A. Caldwell (i) https://orcid.org/0000-0003-1963-9616

Karen A. Collins (1) https://orcid.org/0000-0001-6588-9574

Diana Dragomir (iD https://orcid.org/0000-0003-2313-467X

Michael Fausnaugh (10 https://orcid.org/0000-0002-9113-7162

Ana Glidden (iD https://orcid.org/0000-0002-5322-2315

Joshua E. Schlieder (10 https://orcid.org/0000-0001-5347-7062

Joseph D. Twicken (1D https://orcid.org/0000-0002-6778-7552

Bill Wohler (1) https://orcid.org/0000-0002-5402-9613

\section{References}

Agol, E., \& Deck, K. 2016, ApJ, 818, 177

Astropy Collaboration, Price-Whelan, A. M., Sipőcz, B. M., et al. 2018, AJ, 156,123

Astropy Collaboration, Robitaille, T. P., Tollerud, E. J., et al. 2013, A\&A, 558, A33
Barclay, T., Pepper, J., \& Quintana, E. V. 2018, ApJS, 239, 2

Boyajian, T. S., von Braun, K., van Belle, G., et al. 2012, ApJ, 757, 112

Bradley, L., Sipőcz, B., Robitaille, T., et al. 2019, astropy/photutils: v0.7.2, vv0.7.2, Zenodo, doi:10.5281/zenodo.3568287

Demory, B. O., Gillon, M., Deming, D., et al. 2011, A\&A, 533, 533

Dragomir, D., Teske, J., Günther, M. N., et al. 2019, ApJL, 875, L7

Ducati, J. R. 2002, yCat, 2237, 0

Folsom, C. P., Fossati, L., Wood, B. E., et al. 2018, MNRAS, 481, 5286

Foreman-Mackey, D., Hogg, D. W., Lang, D., \& Goodman, J. 2013, PASP, 125,306

Fortney, J. J., Marley, M. S., \& Barnes, J. W. 2007, ApJ, 659, 1661

Gaia Collaboration 2018, yCat, 1345, 0

Gaia Collaboration, Brown, A. G. A., Vallenari, A., et al. 2018, A\&A, 616, A1

Gaia Collaboration, Prusti, T., de Bruijne, J. H. J., et al. 2016, A\&A, 595, A1

Gangestad, J. W., Henning, G. A., Persinger, R. Y. R., \& Ricker, G. R. 2013, arXiv: 1306.5333

Gillon, M., Demory, B.-O., Benneke, B., et al. 2012, A\&A, 539, A28

Gillon, M., Demory, B.-O., van Grootel, V., et al. 2017, NatAs, 1, 0056

Günther, M. N., \& Daylan, T. 2020, arXiv:2003.14371

Hippke, M., \& Heller, R. 2019, A\&A, 623, A39

Huang, C. X., Quinn, S. N., Vanderburg, A., et al. 2020, ApJL, 892, L7

Huang, C. X., Shporer, A., Dragomir, D., et al. 2018, arXiv:1807.11129

Jenkins, J. M. 2015, AAS Meeting, 3, 106.05

Jenkins, J. M., Twicken, J. D., McCauliff, S., et al. 2016, Proc. SPIE, 9913, 99133E

Johnson, M. C., Endl, M., Cochran, W. D., et al. 2016, ApJ, 821, 74

Knapp, M., Seager, S., Demory, B.-O., et al. 2020, AJ, 160, 23

Knutson, H. A., Charbonneau, D., Allen, L. E., et al. 2007, Natur, 447, 183

Kreidberg, L. 2015, PASP, 127, 1161

Krishnamurthy, A. 2020, PhD Thesis, Massachusetts Institute of Technology

Lissauer, J. J., Fabrycky, D. C., Ford, E. B., et al. 2011, Natur, 470, 53

Maxted, P. F. L. 2016, A\&A, 591, A111

Motalebi, F., Udry, S., Gillon, M., et al. 2015, A\&A, 584, A72

Oja, T. 1993, A\&AS, 100, 591

Pong, C. 2018, in 32nd Annual AIAA/USU Conf. on Small Satellites (Logan, UT: SmallSat), SSC18-PI-34, https://digitalcommons.usu.edu/cgi/viewcontent.cgi? article $=4173 \&$ context $=$ smallsat

Pong, C., Lim, S., Smith, M. W., et al. 2010, Proc. SPIE, 7731, 77311V

Rein, H., \& Liu, S. F. 2012, A\&A, 537, A128

Ricker, G. R., Winn, J. N., Vanderspek, R., et al. 2015, JATIS, 1, 014003

Schwarz, G. 1978, AnSta, 6, 461

Seager, S., Kuchner, M., Hier-Majumder, C. A., \& Militzer, B. 2007, ApJ, 669,1279 
Smith, J. C., Stumpe, M. C., van Cleve, J. E., et al. 2012, PASP, 124, 1000 Smith, M. W., Donner, A., Knapp, M., et al. 2018, in 32nd Annual AIAA/USU Conf. on Small Satellites (Logan, UT: SmallSat), SSC18-I-08, https:// digitalcommons.usu.edu $/ \mathrm{cgi} /$ viewcontent .cgi?article $=4067 \&$ context $=$ smallsat

Smith, M. W., Seager, S., Pong, C. M., et al. 2010, Proc. SPIE, 7731, 773127 Stassun, K. G., Oelkers, R. J., Pepper, J., et al. 2018, AJ, 156, 102

Stumpe, M. C., Smith, J. C., Catanzarite, J. H., et al. 2014, PASP, 126, 100 Stumpe, M. C., Smith, J. C., van Cleve, J. E., et al. 2012, PASP, 124, 985
Sullivan, P. W., Winn, J. N., Berta-Thompson, Z. K., et al. 2015, ApJ, 809, 77 van der Walt, S., Colbert, S. C., \& Varoquaux, G. 2011, CSE, 13, 22

Vanderburg, A., Huang, C. X., Rodriguez, J. E., et al. 2019, ApJL, 881, L19 Virtanen, P., Gommers, R., Oliphant, T. E., et al. 2020, NatMe, 17, 261

Vogt, S. S., Burt, J., Meschiari, S., et al. 2015, ApJ, 814, 12

West, R. G., Gillen, E., Bayliss, D., et al. 2019, MNRAS, 486, 5094

Winn, J. N., \& Fabrycky, D. C. 2015, ARA\&A, 53, 409

Zeng, L., Sasselov, D. D., \& Jacobsen, S. B. 2016, ApJ, 819, 127 